\title{
Insights into the Charge-Transfer Mechanism of Organic Photovoltaics: Effect of Domain Size
}

\author{
Gabriele Boschetto, ${ }^{\dagger}$ Michal Krompiec, ${ }^{\ddagger}$ and Chris-Kriton Skylaris ${ }^{*} \dagger$ \\ $†$ School of Chemistry, University of Southampton, Highfield, Southampton SO17 1BJ, \\ United Kingdom \\ $\ddagger$ Merck Chemicals Ltd., Chilworth Technical Centre, University Parkway, Southampton \\ SO16 7QD, United Kingdom \\ E-mail: c.skylaris@soton.ac.uk \\ Phone: +44 (0)2380 599381
}




\begin{abstract}
A great effort has been devoted into understanding the mechanisms of charge generation and charge separation processes in bulk heterojunction solar cells, with the aim of improving their performance. Theoretical methods, such as DFT, have been used to shed light into these complex processes, but the computational cost associated with the simulations limits the model size, and thus its accuracy with respect to real heterojunctions. To overcome this limitation, a linear-scaling reformulation of (TD)DFT is employed, allowing to move beyond the simple polymer-fullerene models and to consider larger complexes composed of more than a single oligomer chain and numerous fullerene molecules. In this work the interaction between an analogue of PBTZT-stat-BDTT-8, a high-performance D-A statistical copolymer developed by Merck, and PCBM is explored, with a focus on: (i) the effect of the size of the polymer's acceptor (A) blocks, and (ii) the effect of the domain size. Results suggest that large acceptor blocks enhance the probability of a charge transfer (CT) to occur, and that CT states are more significantly affected by the size of the polymer rather than the fullerene phase. Evidence of long-range CT states in the low energy part of the excited state manifold is also observed.
\end{abstract}

\title{
Introduction
}

Organic photovoltaic (OPV) devices have attracted considerable interest in the last 15-20 years, mainly due to their relatively low manufacturing costs, high flexibility, and the possibility of roll-to-roll processing, which enables high and cheap throughput device production; moreover, OPVs also work in both diffuse light and indoor, making them extremely promising as a light harvesting technology. ${ }^{1,2}$

Schematically, a typical OPV module consists of an active layer sandwiched between an anode and a cathode, and composed of a blend of electron donor and electron acceptor materials. This kind of device architecture, which at present is the state of the art in OPVs, is 


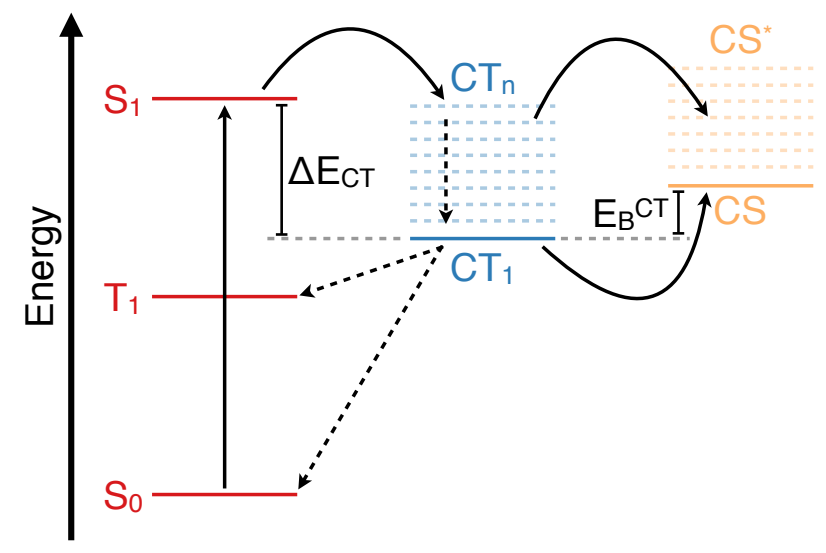

Figure 1: Energy level diagram of the photoinduced charge-carrier formation mechanism in OPVs. $\Delta E_{\mathrm{CT}}$ is defined as the driving force for charge separation, and $E_{\mathrm{B}}^{\mathrm{CT}}$ is the charge transfer binding energy, i.e., the energy required for the hole-electron pair to split and to form the CS state.

known as the bulk heterojunction (BHJ), first reported by Yu et al. in $1995 .{ }^{3}$ In BHJ devices the donor material is typically a $\pi$-conjugated polymer, ideally with a good charge carrier mobility, while the acceptor material is often a fullerene derivative, e.g., phenyl-C61-butyric acid methyl ester $\left(\mathrm{PC}_{61} \mathrm{BM}\right.$ or simply $\left.\mathrm{PCBM}\right)$.

Current understanding of the photoinduced charge-carrier formation mechanism in OPV consists of four fundamental steps, and it is summarized in Figure 1. Initially, the donor material lies in its ground singlet state $\left(\mathrm{S}_{0}\right)$ and through photoabsorption an exciton (one bound electron-hole pair) is formed, generating the donor's first singlet excited state $\mathrm{S}_{1}$ (step i). The exciton diffuses within the polymer bulk and eventually reaches the donor-acceptor interface (step ii), where it may separate into free charges through an intermolecular charge transfer (CT) from the donor to the acceptor material (step iii). Upon CT, the transferred electron gives rise to a 'hot' interfacial and still bound CT state that can relax into a 'cold' $\mathrm{CT}$ state $\left(\mathrm{CT}_{1}\right)$; note that this can undergo a rather fast spin mixing $\left({ }^{1} \mathrm{CT}_{1} \rightleftarrows{ }^{3} \mathrm{CT}_{1}\right)$. The interfacial CT state can now recombine or dissociate: in the first case, depending on the state spin, the electron and the hole can recombine to form the donor ground state, $\mathrm{S}_{0}$, or decay into the donor triplet exciton state, $\mathrm{T}_{1}$; in the second case, if the hole-electron Coulomb attraction is overcome, then the charge separated (CS) state is generated (step 
iv). Charge separation may also happen via coupling of excited $\mathrm{CT}_{n}$ states with the CS state, although at present it is still not clear whether these 'hot' states, as well as the excess energy (i.e., the energy dissipated during the $\mathrm{S}_{1} \rightarrow \mathrm{CT}_{1}$ relaxation process), are important for charge generation: on the one hand, it has been argued that 'hot' CT states, being in general more delocalized, are useful for charge separation because of the significant decrease in the Coulomb interaction between the electron and the hole, and that a large excess energy is therefore beneficial for an efficient charge dissociation; ${ }^{4-7}$ on the other hand, this view has been challenged by experiments that showed that the efficiency of the charge generation step does not depend on the population of high energy $\mathrm{CT}_{n}$ states, but only on the lowest lying one $\left(\mathrm{CT}_{1}\right)$, and therefore, excess energy is regarded as being wasted. ${ }^{8-10}$ The overall power conversion efficiency (PCE) of the device largely depends on the efficiency of steps (iii) and (iv), but due to the morphology of the active layer, an interpenetrating network of polymer and fullenere domains, it is not surprising for many energy loss mechanisms to take place: for instance, the electron-hole pair can undergo geminate recombination even before it is able to diffuse to the donor-acceptor interface, and even assuming the CT to happen, there may occur non-geminate recombination between electrons and holes generated from different excitons. Nevertheless, devices with PCEs higher than 10\% have been widely reported in the literature. ${ }^{11-13}$

Being able to model the formation of charge transfer states in the polymer-fullerene interface would be extremely helpful in order to elucidate the mechanisms of charge generation and separation in OPVs. However, constructing such theoretical models is a rather challenging task, due to the complexity of the system: for instance, although atomistic models based on first-principles calculations (such as density functional theory) would provide some of the necessary information useful to shed light into these highly complex problems, it is not straightforward to correctly represent a BHJ, one of the main reasons being the computational cost associated with the size of the system in study. For this reason, BHJs have often been modelled with DFT mainly as a single small chain oligomer complex with a single 
fullerene. DFT, in its time-dependent extension (TDDFT), has been used to study excited state properties of this type of materials: for instance, Few et al. ${ }^{14}$ investigated the influence of the chemical structure on the properties of CT states of a series of polymer-fullerene complexes, while Niedzialek et al. ${ }^{15}$ tried to correlate the device efficiencies with the excited state properties of three different polymer-fullerene blends, with a focus on the effect of the excess energy; TDDFT has also been used in conjunction with Ehrenfest dynamics for the study of the charge transfer dynamics of a P3HT:PCBM blend. ${ }^{16}$ Recently, Fazzi et al. ${ }^{17}$ explored the influence of the P3HT-PCBM relative orientation (on-top and on-edge) on the excited state manifold of this class of materials, and showed that this is able to affect the charge generation mechanism of OPV blends. However, TDDFT calculations become extremely prohibitive when performed on large-size systems, and this limitation might lead to an oversimplification of the BHJ model. The aim of this work is to move beyond the simple small oligomer and fullerene pair model by increasing the complexity of the system (i.e., by significantly increasing the size of both the oligomer and the fullerene domains), while remaining within the fully ab initio (TD)DFT framework: this is achieved by the use of a linear-scaling reformulation of (TD)DFT, which enables to investigate the ground and excited state properties (with a focus on the charge transfer states) of BHJs on a far larger scale than possible before.

We chose to model the electron acceptor material as PCBM and the electron donor material as an analogue of PBTZT-stat-BDTT-8, a high-performance polymer developed by Merck that was used in the solar tree installation at the German pavilion, as part of the Universal Exhibition in Milan, 2015. ${ }^{18}$ PBTZT-stat-BDTT-8 is a statistical push-pull block copolymer, and this means: (i) it is itself composed of electron-deficient (acceptor, A) and electron-rich (donor, D) blocks, 2,1,3-benzothiadiazole-thiophene (BTZ-T) and benzo-[1,2$b: 4,5$ - $b$ ']dithiophene-thiophene (BDT-T), respectively; (ii) the length of the A and D blocks is not likely to be constant throughout the polymer chain, as this material presents different properties when compared to its regioregular analogues. ${ }^{19}$ Moreover, given its complex com- 
position, it is possible that the size of the blocks may somehow influence the excited state properties of this kind of blend.

In this work we first explore the effect of the polymer's block size by coupling three oligomers, derived from PBTZT-stat-BDTT-8, with a different A block length together with a pristine PCBM supercell, focusing on the charge transfer excitations of the resulting complexes. We then multiply in turn the size of the fullerene and the polymer domains (resulting in models with as many as 2360 and 2048 atoms, respectively), in order to investigate how far the charge transfer states can delocalize in both domains, how (and if) CT states are influenced by the domain size, and to elucidate whether long-range charge transfer (i.e.,

charge transfer states with an increased electron-hole distance) might occur in OPV devices, as the work of Ma et al. ${ }^{20}$ seems to suggest.

This study has been carried out within the density functional theory framework by employing linear-scaling DFT and linear-scaling TDDFT with the ONETEP code, and it is organized as follows: in Section 2 (Methods) the concepts behind the ONETEP program, the setup details of the simulations and a benchmark of charge transfer calculations with TDDFT are presented; in Section 3 (Results and Discussion) results are shown and their implications for organic photovoltaics are discussed. The conclusions are then presented in Section 4 (Conclusion).

\section{Methods}

\section{The ONETEP program}

All the simulations were performed at the density functional theory level by using the ONETEP (Order-N Electronic Total Energy Package) code for linear-scaling DFT. ${ }^{21}$ ONETEP is based on a reformulation of the single-particle density matrix $\rho\left(\mathbf{r}, \mathbf{r}^{\prime}\right)$, which in conventional 
Kohn-Sham DFT is defined as

$$
\rho\left(\mathbf{r}, \mathbf{r}^{\prime}\right)=\sum_{i} f_{i} \psi_{i}(\mathbf{r}) \psi_{i}^{*}\left(\mathbf{r}^{\prime}\right),
$$

where $f_{i}$ is the occupancy of the state $\psi_{i}(\mathbf{r})$, which is a Kohn-Sham orbital function. In the ONETEP formalism, the density matrix is expressed in a separable form, equivalent to the expression in Equation 1:

$$
\rho\left(\mathbf{r}, \mathbf{r}^{\prime}\right)=\sum_{\alpha \beta} \phi_{\alpha}(\mathbf{r}) K^{\alpha \beta} \phi_{\beta}^{*}\left(\mathbf{r}^{\prime}\right),
$$

where $\phi_{\alpha}(\mathbf{r})$ are localized atom-centered orbital functions known as non-orthogonal generalised Wannier functions (NGWFs), ${ }^{22}$ and $K$ is known as the density kernel. The NGWFs are expanded in a basis of periodic sinc (psinc) functions, ${ }^{23}$ which correspond to plane waves through a Fourier transform. The peculiarity of ONETEP is that it is able to achieve linearscaling behavior while maintaining at the same time near-complete basis set accuracy: linear scaling is obtained by enforcing strict localization of the NGWFs within a set radius and by truncation of the density kernel, $K$, imposing a spatial cutoff, which makes $\rho(\mathbf{r}, \mathbf{r}$ ') sparse; during a calculation both the density kernel and the NGWFs are optimized in a self-consistent way, and this allows plane wave accuracy by using a minimal set of orbital functions only.

\section{Simulation Details}

In this work the ground and excited state properties of a donor-acceptor model complex have been studied (see Figure 2 for the structures of the materials); in order to avoid confusion with the electron rich and electron poor units of the polymer itself, from this point onwards the donor material will be simply called polymer $(\mathrm{P})$ and the acceptor material fullerene (F). Also note that, although the name PBTZT-stat-BDTT-8 will still be used, we here

emphasize that the structures that were used to carry out this study were all analogues of this polymer. In this study the fullerene domain was modelled as twelve close-packed PCBM molecules (effectively a $3 \times 1 \times 1$ supercell) whose atomic positions were taken from a pristine 


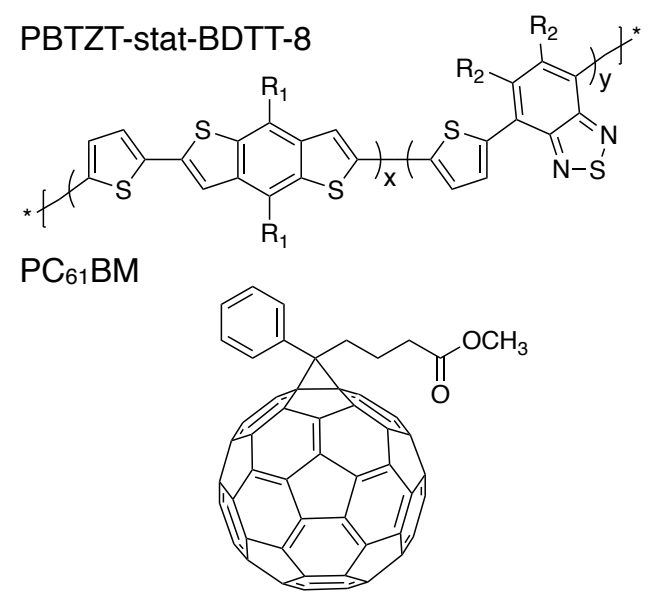

Figure 2: Structure of PCBM and the repeat unit of PBTZT-stat-BDTT-8 analogue, with $x$ being the BDT-T block (donor, D) and $y$ the BTZ-T block (acceptor, A). In this work, the $\mathrm{R}_{1}$ and $\mathrm{R}_{2}$ functional groups of the polymer are $\mathrm{CH}_{3}$ and $\mathrm{OCH}_{3}$, respectively.

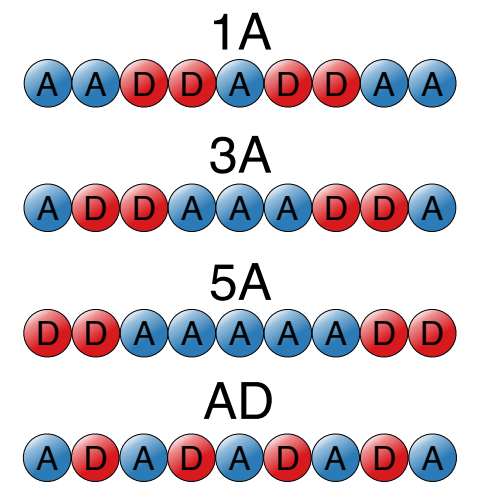

Figure 3: Schematic representation of the 1A, 3A, 5A, and AD oligomer structures. Donor units are shown in red, while acceptor units in blue. 
PCBM crystal experimentally determined from XRD by Paternò et al. ${ }^{24}$

We first focused on the effect of the A block length on the properties of the resulting polymer:fullerene complex, since, as previously mentioned, this might or might not be important for polymer design considerations. It is worth noting that our previous studies suggested different electronic and optical properties of the polymer's D and A units when blended with PCBM (see Supporting Information). To explore the effect of the A block length, the D and A units of PBTZT-stat-BDTT-8 were combined so that three different oligomers were obtained: (i) DDAAAAADD, (ii) ADDAAADDA, and (iii) AADDADDAA; for comparison with the regioregular copolymer, a fourth oligomer was also constructed, the alternating ADADADADA. The oligomers were called 5A, 3A, 1A, and AD, respectively (see Figure 3 for a schematic representation of these structures). It is worth noting that the oligomers mentioned above are all isomers. The PCBM supercell was placed at the center of each oligomer chain, above the central A block, at an initial intermolecular distance of $\sim 3.5 \AA$, in line with other theoretical works in which the distance was set between 3.2 and $3.5 \AA .{ }^{14-16,25}$ The size of the A block was gradually reduced by taking away at each step two A units from the central block and by placing them at the edge of the chain: in this way the number of A and D blocks, the length of the chains, the number of atoms and double bonds were kept constant for each simulation, assuring the results to be comparable; the assumption made behind these experiments is that the external A units are too far away from the central fullerene domain to be able to significantly interact with it. Each of the complexes consists of a total of 1304 atoms.

For the final, and main part of this study we focused on the 5A complex and we increased the complexity of the system: we multiplied in turn the size of the fullerene domain (from a $3 \times 1 \times 1$ to a $3 \times 1 \times 2$ supercell) and the size of the polymer domain (two, three, four stacked oligomer chains), in order to investigate how far the charge transfer states can extend in the PCBM crystal and in the polymer domain, respectively, and if this has any effect on the interface energetics. The geometries of the stacked oligomers were first fully optimized 
without the presence of the fullerene phase, and we set the interchain distance at a value of $\sim 3.5 \AA$. The chains were stacked in such a way that each oligomer was flipped by $180^{\circ}$ with respect to the closest ones above and below, with the functional groups pointing in opposite directions, in order to avoid steric hindrance. We then placed the PCBM phase above the central part of the polymer domain, and we fully optimized each one of the complexes.

The geometries of all oligomers, fullerenes, and polymer-fullerene complexes were optimized in vacuum by using the Perdew-Burke-Ernzerhof exchange-correlation functional $(\mathrm{PBE})^{26}$ in conjunction with the projector augmented wave (PAW) method; ${ }^{27}$ to take into account dispersion effects, each simulation was performed also by including the D2 correction by Grimme. ${ }^{28}$ Single-point energy calculations were carried out by using the PBE functional with norm-conserving pseudopotentials to model core electrons. After calibration of the method, optimal values for the kinetic energy cutoff and the NGWFs radii were found to be $800 \mathrm{eV}$ and $9.0 \mathrm{bohr}$, respectively; a number of 4 NGWFs per atom was chosen for $\mathrm{C}$, $\mathrm{N}, \mathrm{O}, \mathrm{S}$, and $1 \mathrm{NGWF}$ was assigned to each $\mathrm{H}$ atom. To model excited state properties in polymer:fullerene blends, linear-scaling time-dependent DFT (TDDFT), as implemented in the ONETEP code in its linear response formalism, was used. ${ }^{29,30}$ TDDFT calculations were performed in vacuum with the PBE functional (at present, only nonhybrid functionals are available in ONETEP for time-dependent simulations), and within the Tamm-Dancoff approximation (TDDFT/TDA), ${ }^{31}$ which is known to work reasonably well for valence-excited states, and yields identical results with respect to conventional TDDFT for CT excitations, ${ }^{32}$ while making the calculations less computationally costly. In order to perform TDDFT with ONETEP, a second set of NGWFs needs to be optimized so that also the conduction states, which are more delocalized and lie at higher energies, can be correctly represented: ${ }^{33}$ the valence and conduction NGWFs are combined to form a joint set of NGWFs that allows an efficient representation of excited states for TDDFT calculations. For the conduction set, a number of 13 NGWFs for $\mathrm{C}, \mathrm{N}, \mathrm{O}, \mathrm{S}$ and $5 \mathrm{NGWFs}$ for $\mathrm{H}$ atoms was chosen, with a radius of $12.0 \mathrm{bohr}$. 
Describing charge transfer states with TDDFT is a rather challenging task, since this is an area in which this method has well known limitations due to both the DFT formalism itself (i.e., the missing derivative discontinuity of the exchange-correlation functional), and the incorrect $1 / R$ behavior of the potential energy curves of such states, with $R$ being the intermolecular separation distance. These both lead to one major drawback: an underestimate of the energy of the $\mathrm{CT}$ transitions, and consequently the loss of a direct quantitative comparison with experimental values. This is in general a significant issue when using a GGA functional, such as PBE, and it might in part be corrected (but not necessarily fully solved) by employing functionals which include a fraction of Hartree-Fock exchange, such as hybrids. Range-separated hybrids instead seem to work particularly well for describing CT states, however, they are more computationally expensive, and they introduce an empirical parameter that significantly affects the excitation energies, and that therefore needs to be tuned. Taking these into account, we note that qualitative results that can be useful to shed light into some of the properties of polymer-fullerene complexes can still be obtained despite the use of a GGA functional, and moreover, to the best of our knowledge, DFT and TDDFT have never been applied so far to such large systems which are relevant for OPV functionality. To assess TDDFT accuracy, this methodology was first tested by comparing results obtained with ONETEP against published theoretical and experimental data. A number of eight polymer-fullerene blends were considered, of which the experimental CT peak emissions obtained with electroluminescence (EL) were taken from the work of Yao et al. ${ }^{34}$ Amongst these eight blends, five of them were also modelled with TDDFT by Few et al.: ${ }^{14}$ as previously mentioned, in their work they performed TDDFT to model the first CT excitation energies of different blends at the B3LYP/6-31G* level (for further details the reader is referred to their paper). In this paper the blends were modelled by considering oligomers consisting of 3-4 repeat units (12 in the case of P3HT, due to the very small size of its repeat unit), and by placing the fullerene (either $\mathrm{PC}_{61} \mathrm{BM}$ or $\mathrm{PC}_{71} \mathrm{BM}$ ) above the acceptor unit of the oligomer, with the only exception of P3HT, the latter being a homopolymer. Note that 
for every complex, the fullerene was placed above the central A unit, where present, of the oligomer chain. Results are shown in Figure 4: as can be observed, and as expected, a GGA functional like PBE underestimates the energies of the $\mathrm{CT}$ states, while the hybrid functional B3LYP has the opposite effect; as Few et al. pointed out in their work, this overestimate with the B3LYP functional is most probably due to the lack of polarizable medium and to the use of a rather limited basis set. Despite the expected systematical underestimate of the frequency of $\mathrm{CT}_{1}$ states, results seem to suggest that $\mathrm{PBE}$ is able to at least qualitatively represent the overall experimental trend, with the only exception of MDMO-PPV:PC ${ }_{61} \mathrm{BM}$; however, as it can be observed, also B3LYP seems to fail for this particular blend. Therefore, it seems that by using a less computationally expensive functional such as PBE, it is possible to compute charge transfer excitation energies that overall agree qualitatively with experiments, at least with this class of systems.

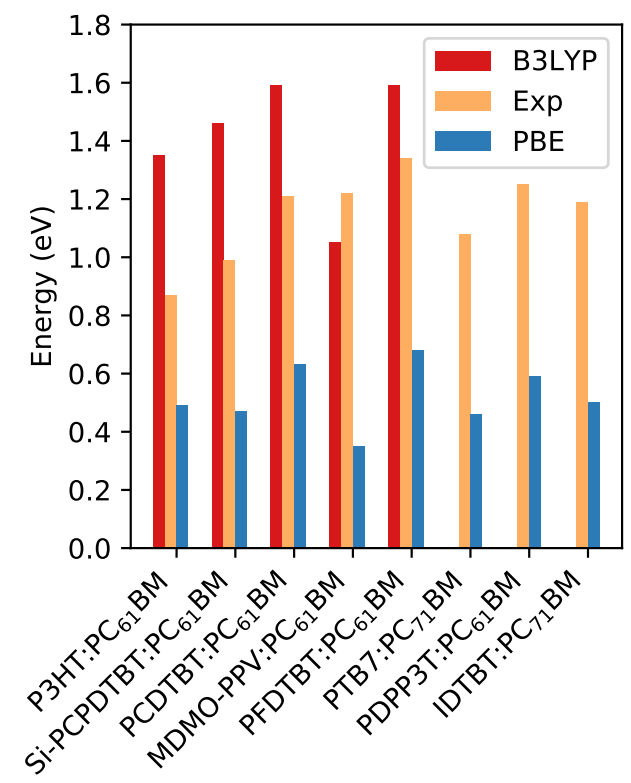

Figure 4: Comparison between experimental (from Ref. 34) and theoretical first charge transfer excitations $\left(\mathrm{CT}_{1}\right)$ of a series of polymer-fullerene blends. Theoretical values were computed with ONETEP at the PBE level, while B3LYP results are estimated from Ref. 14 .

The experimental CT state energy of PBTZT-stat-BDTT-8:PC ${ }_{61} \mathrm{BM}$ blend is $\sim 1.3 \mathrm{eV}$, as estimated by EQE measurements carried out at Merck. We note that this value is also in 
accord with the empirical relation found by Benduhn et al. ${ }^{35}$ which states that the difference between $E_{\mathrm{CT}}$ and $q V_{\mathrm{OC}}$ (open-circuit voltage) is, in general, constant for a wide range of materials and equal to $0.5-0.6 \mathrm{eV}$ : the $V_{\mathrm{OC}}$ of PBTZT-stat-BDTT-8: $\mathrm{PC}_{61} \mathrm{BM}$ is in fact $\sim 0.8$ V. In order to overcome the GGA systematic underestimate of CT energies and to rescale our absorption spectra to match experiments, we applied a constant shift factor of 1.08 $\mathrm{eV}$ to our theoretical PBE absorption spectra; we calculated this factor by subtracting the energy of the $S_{1}$ transition of the system composed of four stacked oligomer chains (without the fullerene phase) computed at the TDDFT/PBE level to the experimental optical gap of PBTZT-stat-BDTT-8 $(1.75 \mathrm{eV})$. Note, however, that when computing properties such as complex binding energies $\left(E_{\mathrm{B}}\right)$ and charge transfer exciton binding energies $\left(E_{\mathrm{B}}^{\mathrm{CT}}\right)$, no shift was applied. Polymer-fullerene complex binding energy is defined as:

$$
E_{\mathrm{B}}=E^{\mathrm{PF}}-\left(E^{\mathrm{P}}+E^{\mathrm{F}}\right),
$$

where $E^{\mathrm{PF}}$ is the energy of the whole complex, and $E^{\mathrm{P}}$ and $E^{\mathrm{F}}$ correspond to the energy of the polymer and the fullerene phase, respectively. $E_{\mathrm{B}}^{\mathrm{CT}}$ was estimated according to the following expression: ${ }^{36}$

$$
E_{\mathrm{B}}^{\mathrm{CT}}=E_{\mathrm{CS}}-E_{\mathrm{CT}_{1}}=(\mathrm{IP}-\mathrm{EA})-E_{\mathrm{CT}_{1}},
$$

where $E_{\mathrm{CS}}$ is the energy of the charge separated state, and $E_{\mathrm{CT}_{1}}$ is the energy of the first charge transfer state; as shown by Brédas et al. ${ }^{37}$ the energy of the CS state can be estimated as the difference between the ionization potential (IP) and the electron affinity (EA) of the complex. 


\title{
Results and Discussion
}

\author{
Effect of the A block length
}

\section{Side view}

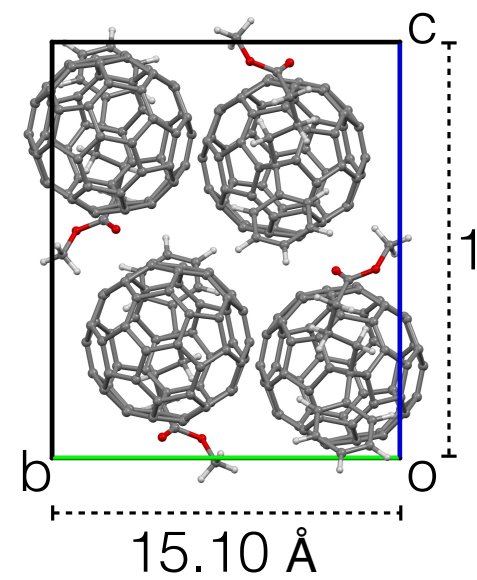

Front view

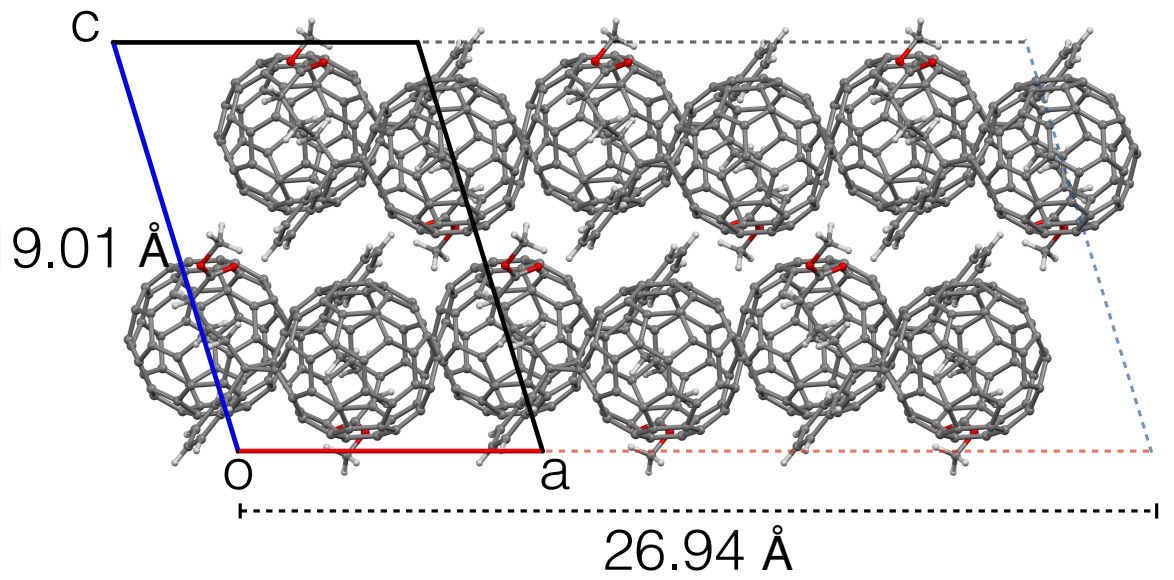

Figure 5: Structure and cell parameters of the 3x1x1 PCBM supercell, derived from X-ray diffraction (see Ref. 24).

Table 1: Breakdown of the first ten CT states for PCBM:5A, PCBM:3A, PCBM:1A and PCBM:AD complexes. ${ }^{a}$

\begin{tabular}{|c|c|c|c|c|}
\hline $\mathrm{CT}$ & PCBM:5A & PCBM:3A & PCBM:1A & PCBM:AD \\
\hline 1 & $\mathrm{HOMO} \rightarrow$ LUMO & $\mathrm{HOMO} \rightarrow$ LUMO & $\mathrm{HOMO} \rightarrow \mathrm{LUMO}$ & $\mathrm{HOMO} \rightarrow$ LUMO \\
\hline 2 & $\mathrm{HOMO} \rightarrow \mathrm{LUMO}+1$ & $\mathrm{HOMO} \rightarrow \mathrm{LUMO}+1$ & $\mathrm{HOMO} \rightarrow \mathrm{LUMO}+1$ & $\mathrm{HOMO} \rightarrow \mathrm{LUMO}+1$ \\
\hline 3 & $\mathrm{HOMO} \rightarrow \mathrm{LUMO}+2$ & $\mathrm{HOMO} \rightarrow \mathrm{LUMO}+2$ & $\mathrm{HOMO} \rightarrow \mathrm{LUMO}+2$ & $\mathrm{HOMO} \rightarrow \mathrm{LUMO}+2$ \\
\hline 4 & $\mathrm{HOMO} \rightarrow \mathrm{LUMO}+3$ & $\mathrm{HOMO} \rightarrow \mathrm{LUMO}+3$ & $\mathrm{HOMO} \rightarrow \mathrm{LUMO}+3$ & $\mathrm{HOMO} \rightarrow \mathrm{LUMO}+3$ \\
\hline 5 & $\mathrm{HOMO} \rightarrow \mathrm{LUMO}+4$ & $\mathrm{HOMO} \rightarrow \mathrm{LUMO}+4$ & $\mathrm{HOMO} \rightarrow \mathrm{LUMO}+4$ & $\mathrm{HOMO} \rightarrow \mathrm{LUMO}+4$ \\
\hline 6 & $\mathrm{HOMO} \rightarrow \mathrm{LUMO}+5$ & $\mathrm{HOMO} \rightarrow \mathrm{LUMO}+5$ & $\mathrm{HOMO} \rightarrow \mathrm{LUMO}+5$ & $\mathrm{HOMO} \rightarrow \mathrm{LUMO}+5$ \\
\hline 7 & HOMO- $1 \rightarrow$ LUMO & $\mathrm{HOMO} \rightarrow \mathrm{LUMO}+6$ & $\mathrm{HOMO} \rightarrow \mathrm{LUMO}+6$ & $\mathrm{HOMO} \rightarrow \mathrm{LUMO}+6$ \\
\hline 8 & $\mathrm{HOMO} \rightarrow \mathrm{LUMO}+6$ & $\mathrm{HOMO} \rightarrow \mathrm{LUMO}+7$ & HOMO-1 $\rightarrow$ LUMO & HOMO-1 $\rightarrow$ LUMO \\
\hline 9 & $\mathrm{HOMO} \rightarrow \mathrm{LUMO}+7$ & $\begin{array}{l}0.85(\text { HOMO }-1 \rightarrow \text { LUMO }) \\
0.15(\text { HOMO } \rightarrow \text { LUMO }+8)\end{array}$ & $\mathrm{HOMO} \rightarrow \mathrm{LUMO}+7$ & $\mathrm{HOMO} \rightarrow \mathrm{LUMO}+7$ \\
\hline 10 & $\mathrm{HOMO}-1 \rightarrow \mathrm{LUMO}+1$ & $\mathrm{HOMO} \rightarrow \mathrm{LUMO}+9$ & $\begin{array}{l}0.49(\mathrm{HOMO}-1 \rightarrow \mathrm{LUMO}+1) \\
0.48(\mathrm{HOMO} \rightarrow \mathrm{LUMO}+8)\end{array}$ & $\begin{array}{l}0.70(\mathrm{HOMO} \rightarrow \mathrm{LUMO}+8) \\
0.27(\mathrm{HOMO} \rightarrow \mathrm{LUMO}+9)\end{array}$ \\
\hline
\end{tabular}

electron-donating orbital is located on the oligomer while every electron-accepting orbital is located on the fullerene domain.

The 3x1x1 PCBM supercell was positioned on top of oligomers 1A, 3A, 5A, AD; when the oligomers are coupled with the PCBM phase, we will refer to the corresponding complexes as PCBM:1A, PCBM:3A, PCBM:5A, and PCBM:AD. From a structural point of view, the 


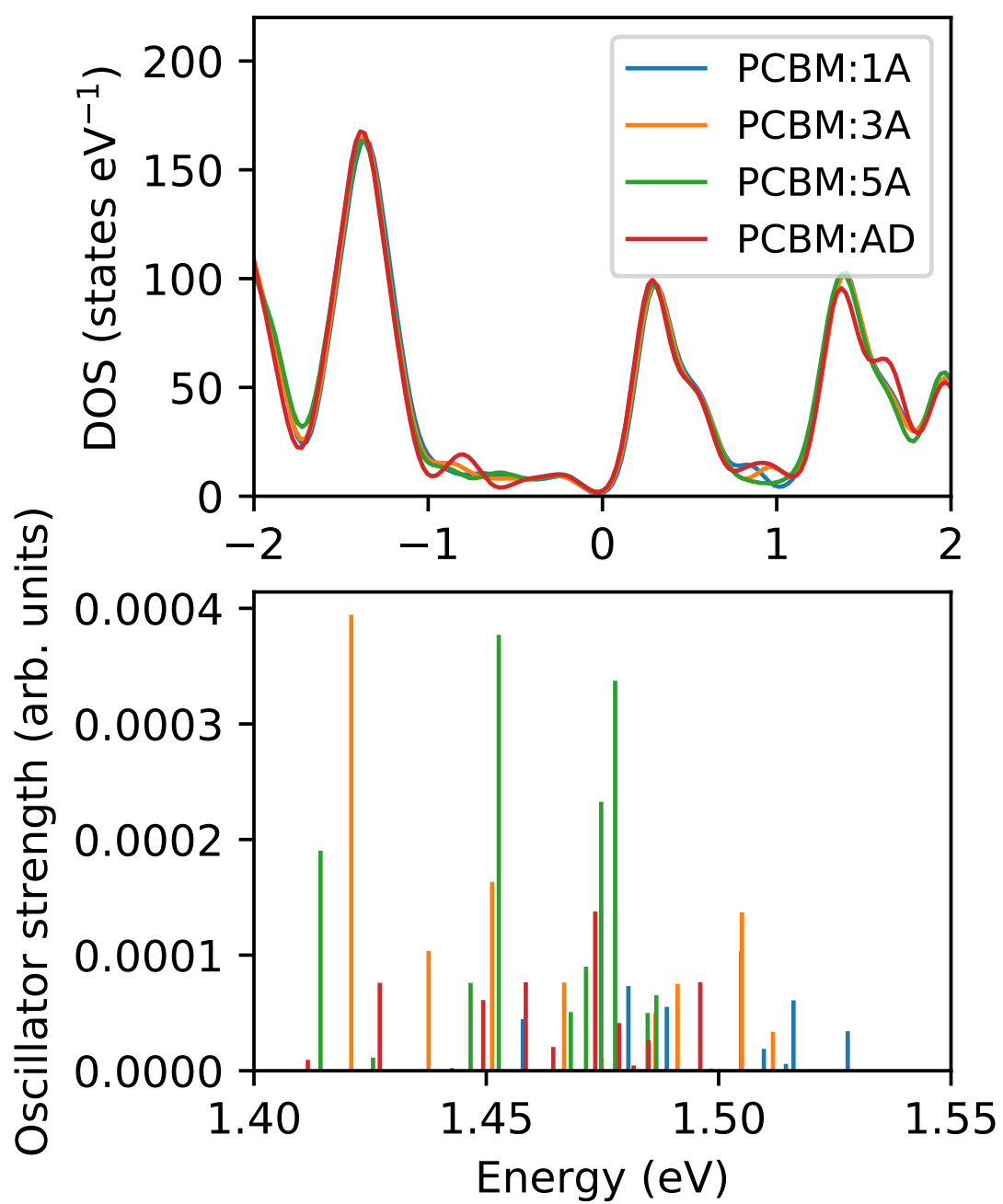

Figure 6: Density of states and excited state spectra of PCBM:5A, PCBM:3A, PCBM:1A, and PCBM:AD complexes. The DOS are centered around the Fermi levels of the systems, and a Gaussian smearing of $0.1 \mathrm{eV}$ was used. Note that in the spectra a shift was applied to correct the values of the transition energies with respect to experiments. 


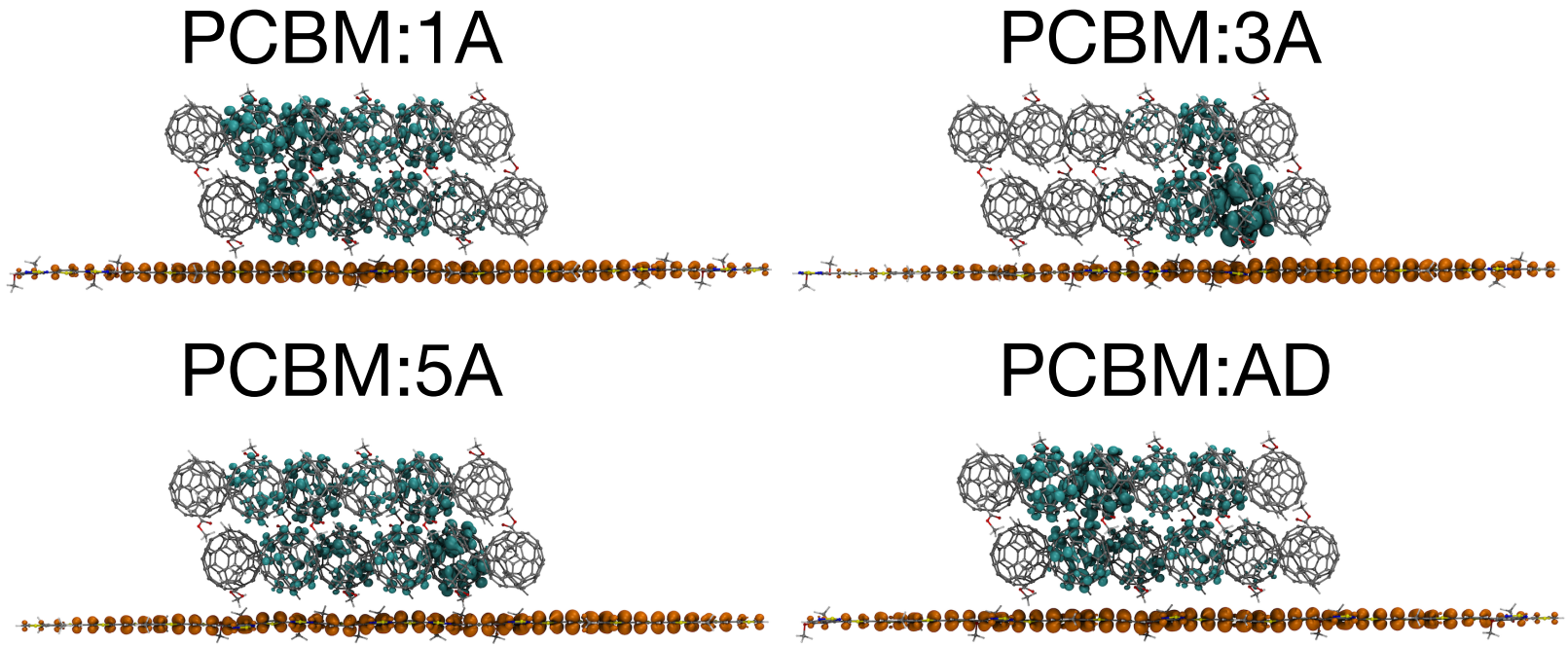

Figure 7: Electron-hole density plots of the first charge transfer states $\left(\mathrm{CT}_{1}\right)$ of $\mathrm{PCBM}: 1 \mathrm{~A}$, PCBM:3A, PCBM:5A, and PCBM:AD complexes. Hole density is shown in orange, while electron density is shown in cyan.

length of the four oligomers is approximately $90.0 \AA$, and the length of the central A block is $7.0 \AA, 23.5 \AA$ and $39.8 \AA$ for $1 \mathrm{~A}, 3 \mathrm{~A}$ and $5 \mathrm{~A}$, respectively. The PCBM $3 \mathrm{x} 1 \mathrm{x} 1$ supercell parameters are $a=26.94 \AA, b=15.10 \AA, c=19.01 \AA$, and $\alpha=90^{\circ}, \beta=106.90^{\circ}, \gamma=90^{\circ}$; the average PCBM nearest neighbour distance is $10.05 \AA$. It is worth noting that it has been experimentally observed that the coherence length of the fullerene phase in BHJs is 1.1-1.8 $\mathrm{nm},{ }^{38}$ and therefore our models are big enough to be representative. The PCBM supercell, together with its parameters, is shown in Figure 5. Results show that the size of the A block does not significantly influence properties such as the distribution of energy levels, the band gaps (0.34-0.36 eV), and the density of states, as can be observed from Figure 6, upper plot. However, we note an interesting trend in the complex binding energies $\left(E_{\mathrm{B}}\right)$ : for PCBM:5A the computed binding energy is $-1.55 \mathrm{eV}\left(-35.74 \mathrm{kcal} \mathrm{mol}^{-1}\right)$, while for both PCBM:3A and PCBM:1A the binding energy is $-1.21 \mathrm{eV}\left(-27.90 \mathrm{kcal} \mathrm{mol}^{-1}\right)$; an intermediate value of $1.35 \mathrm{eV}\left(-31.13 \mathrm{kcal} \mathrm{mol}^{-1}\right)$ is found for PCBM:AD. We observe higher values of $E_{\mathrm{B}}$ with respect to results discussed in the literature by Ravva et al., ${ }^{39}$ and we attribute this effect mainly to the large fullerene domain, where the dispersion forces are significant. This is 
confirmed by decomposing $E_{\mathrm{B}}$ into its electronic and dispersion contributions: in accordance with results by Ravva et al., we find that dispersion forces are the major contribution to the binding energies, and in fact dispersion binding energies are $-1.86 \mathrm{eV}\left(-42.89 \mathrm{kcal} \mathrm{mol}^{-1}\right)$, $-1.59 \mathrm{eV}\left(-36.67 \mathrm{kcal} \mathrm{mol}^{-1}\right),-1.31 \mathrm{eV}\left(-30.21 \mathrm{kcal} \mathrm{mol}^{-1}\right)$, and $-1.46 \mathrm{eV}\left(-33.67 \mathrm{kcal} \mathrm{mol}^{-1}\right)$ for PCBM:5A, PCBM:3A, PCBM:1A, and PCBM:AD, respectively; these results imply a significantly lower electronic repulsion effect with respect to dispersion forces. We note that PCBM:5A has a significantly higher complex binding energy with respect to both PCBM:3A and PCBM:1A $\left(\Delta E_{\mathrm{B}}=0.34 \mathrm{eV}, 7.84 \mathrm{kcal} \mathrm{mol}^{-1}\right)$, and also to PCBM:AD $\left(\Delta E_{\mathrm{B}}=0.20 \mathrm{eV}\right.$, $4.61 \mathrm{kcal} \mathrm{mol}^{-1}$ ); we also note that the dispersion binding energy correlates well with the size of the A block (the larger its size, the stronger the interaction with PCBM): overall, these results show some evidence of a better mixing between the fullerene domain and the A blocks of PBTZT-stat-BDTT-8, as observed in experiments. ${ }^{40}$

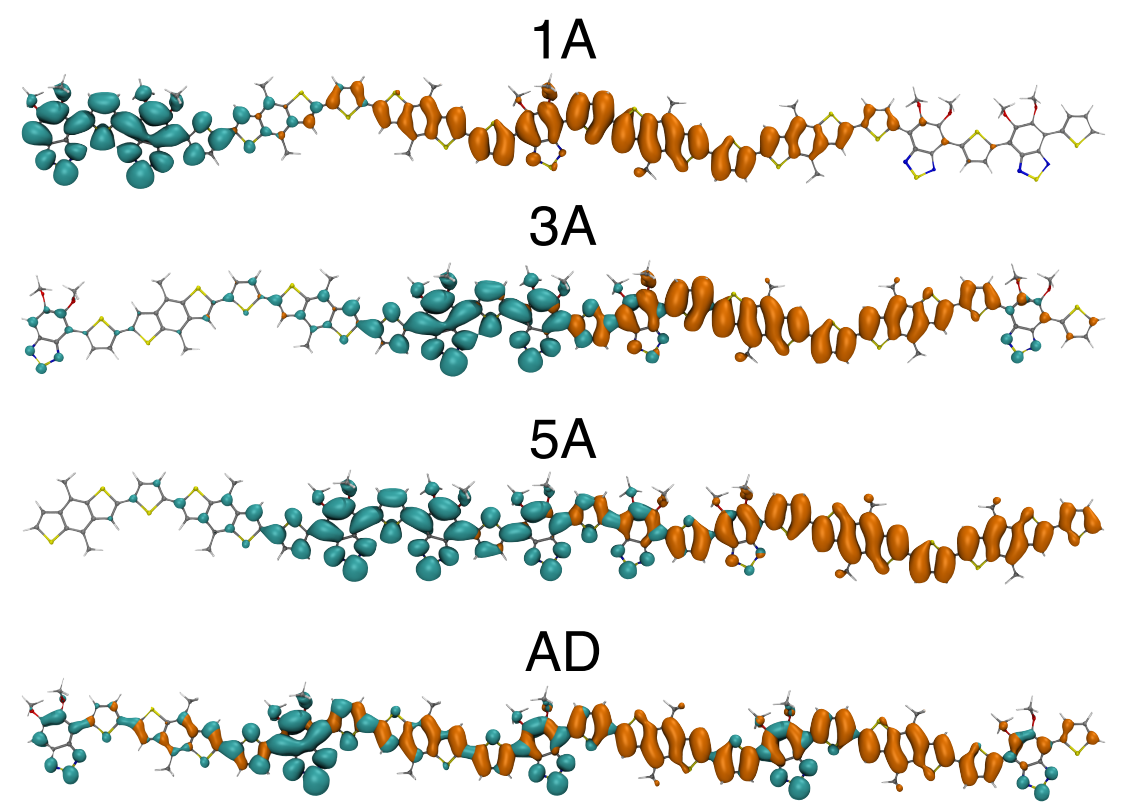

Figure 8: Hole-electron density plots of 1A, 3A, 5A, and AD isolated oligomers. Electron density is shown in cyan, while the hole density in orange.

We then explored the excited state properties by performing TDDFT on each complex, and requiring the calculation of the first ten excited states; results are shown in Figure 6, lower plot, and a breakdown of each transition is presented in Table 1. For each complex 
the first 10 excited states all have a charge transfer character, since each CT state involves transitions between the occupied orbitals of the oligomer and the virtual orbitals of the PCBM domain; the majority of transitions occur from the oligomers' HOMO level, but at higher energies, transitions from the deeper HOMO-1 level are also observed. It is worth noting that the first six CT states do not differ amongst complexes, while this is not true for the remaining states. We also note that the range of energies, corrected by applying the constant shift discussed in the Methods section, in which the CT states lie are in each case similar, between $\sim 1.44 \mathrm{eV}$ and $\sim 1.53 \mathrm{eV}$. Hole-electron density plots of each transition of each complex, some of which are shown in Figure 7, confirm that these are all CT states, since the electron density is located on the fullerene domain, while the hole density on the oligomer chains, as expected. We note here that the electron density of each transition is not strictly localized on a single PCBM molecule, but is delocalized over multiple molecules, implying that during an electronic transition fullerenes do interact with each others and the transferred electron can delocalize on more than a single PCBM. We also observe that the fullerene molecules that interact with the oligomer chain are not necessarily only the closest ones to it. We also computed CT exciton binding energies $\left(E_{\mathrm{B}}^{\mathrm{CT}}\right)$ of the complexes, and they all lie between $\sim 0.6$ and $\sim 0.7 \mathrm{eV}$, not too far from typical experimental values: ${ }^{36}$ more specifically, $0.68 \mathrm{eV}, 0.63 \mathrm{eV}$, and $0.71 \mathrm{eV}$ for PCBM:5A, PCBM:3A, and PCBM:1A, respectively; we found a value of $0.61 \mathrm{eV}$ for PCBM:AD. Although the $E_{\mathrm{B}}^{\mathrm{CT}}$ all lie within a small energy range, we note that PCBM:1A is the complex with the highest charge transfer binding energy, while interestingly PCBM:AD has the lowest $E_{\mathrm{B}}^{\mathrm{CT}}$, comparable to the value obtained for PCBM:3A.

Despite involving similar orbital transitions, the shape of the excited state spectra is different for each complex (see Figure 6, lower plot), and, interestingly, we observe an average decreasing trend in the oscillator strengths with the decrease of the A block size, with the only exception of the first CT transition of PCBM:3A, which is, notably, the brightest one; interestingly, we also observe low oscillator strengths in PCBM:AD. This gradual decrease 
in the oscillator strength may depend on the amount of charge density over the A block of the oligomers: it has been observed that push-pull copolymers undergo intramolecular charge transfer upon photoabsorption, i.e., the charge gets transferred from the D block to the A block within the polymer. ${ }^{41,42}$ Since we decreased the size of the A block $(5 \mathrm{~A}>3 \mathrm{~A}>$ $1 \mathrm{~A}-\mathrm{AD}$ ), it is reasonable to believe that the amount of charge located in the A block will also decrease, effectively lowering the probability of an electronic transition to take place, and consequently switching off the oscillator strength. In order to gain more insight into this matter, we focused on the oligomers alone, and we studied their excited state properties when decoupled from the fullerene domain.

We performed TDDFT on $1 \mathrm{~A}, 3 \mathrm{~A}, 5 \mathrm{~A}$, and $\mathrm{AD}$ oligomers, requiring the calculation of the $\mathrm{S}_{1}$ state only. The hole-electron density plots of each oligomer obtained with TDDFT are shown in Figure 8. Results now confirm the previous hypothesis: it is clear that the extent of the electron density correlates with the length of the central A block of the oligomers, as can be observed. For oligomers $3 \mathrm{~A}$ and $5 \mathrm{~A}$ the electron density is localized onto the central A block and the hole density is found onto the D units, while, interestingly, despite the presence of a single $\mathrm{A}$ unit at the center of the $1 \mathrm{~A}$ oligomer chain, the electron density is localized exclusively at the edge, above the external A block composed of two A units. The hole density extends over the center of the chain, that is the part of the oligomer that in our model will mainly interact with the PCBM domain. For oligomer AD, as expected, the hole density is found on the D units, while the electron density on the A units; we note that most probably it is mainly the central A unit that is able to significantly interact with the fullerene domain, and this explains why PCBM:AD and PCBM:1A transitions have a comparable intensity. This finding seems to suggest that the relative size of different blocks in the polymer may be an important factor, since, assuming the size of the blocks not being constant in the real polymer, when the material undergoes photoexcitation the charge seems to get transferred mainly to the A blocks whose size is bigger or comparable to the $\mathrm{D}$ blocks. This is relevant because in the real blend the polymer most likely contains long A blocks 
rather than long $\mathrm{D}$ blocks. We also note that the energy of the $\mathrm{S}_{1}$ transition is the same for both oligomers $3 \mathrm{~A}$ and $5 \mathrm{~A}(0.93-0.92 \mathrm{eV})$, while for $1 \mathrm{~A}$ the energy is slightly lower $(0.86$ $\mathrm{eV})$, and, on the contrary, oligomer AD has a slightly higher value $(0.98 \mathrm{eV})$. Overall, these results suggest that the size of the A block does indeed have an influence on the excited state properties of both the polymer itself and the PCBM complex: the length of the blocks in the polymer chain determines where the charge is more likely to get transferred to, and if the PCBM domain does not overlap with the portion of the polymer chain where there is a high charge concentration, this will decrease the probability of a charge transfer transition to be observed. We stress here that even if low oscillator strengths correlate with a low probability of electronic transitions to occur, this does not necessary mean that in the actual device a low efficiency will be observed, since this property does not depend exclusively on the energetics of the molecules involved, but for instance both the morphology and the device architecture are crucial. Nevertheless, these results can provide some guidance on how to enhance the charge transfer rate of push-pull polymer-fullerene blends.

\section{Effect of the size of the domain}

Having demonstrated in the previous section that large A blocks are beneficial for charge transfer processes, we chose PCBM:5A complex as the starting model for this final and most relevant part of the paper. From this point onwards the starting model will be called simply P:F (polymer:fullerene), the complexes composed of $n$ oligomer chains (with $n=2,3,4$ ) will be called $n \mathrm{P}: \mathrm{F}$, and the complex with twice the number of PCBM molecules will be called P:2F (the structures of the five complexes are shown in Figure 9). The 3x1x2 PCBM supercell parameters are $a=26.94 \AA, b=15.1 \AA, c=38.02 \AA$, and $\alpha=90^{\circ}, \beta=106.90^{\circ}$, $\gamma=90^{\circ}$; the average PCBM nearest neighbour distance is once again $10.05 \AA$.

Contrary to the results presented in the previous section, the ground state properties of this set of complexes are significantly different, as can be observed from Figure 10, upper plot: for instance, if doubling the fullerene domain does not cause any change in the band 


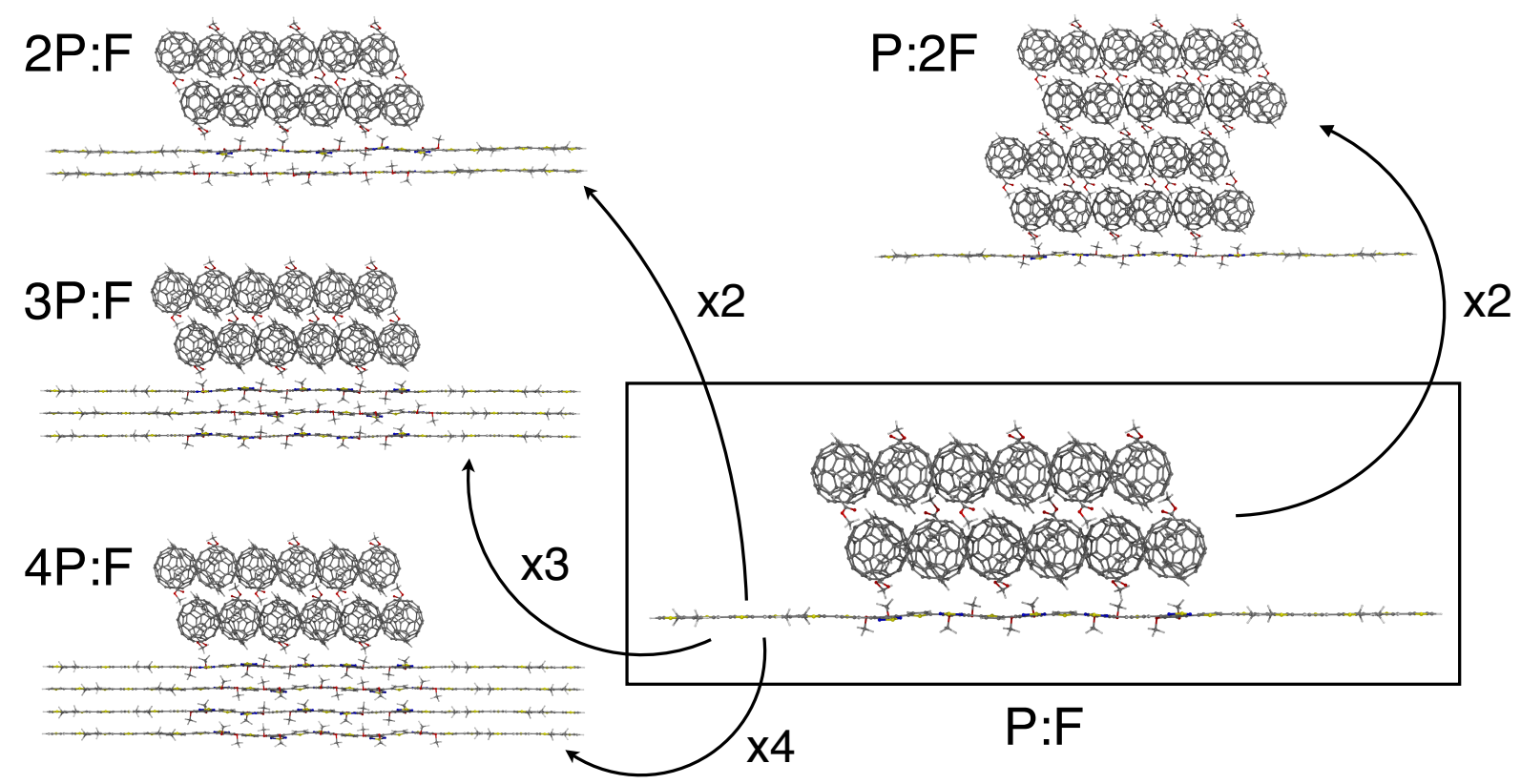

Figure 9: Optimized structures at the PBE-D2 level of P:F, P:2F, 2P:F, 3P:F, and 4P:F complexes.

gap of the complex, it does affect the density of states, which becomes more intense due to the higher number of states in the system. On the contrary, increasing the polymer phase does not cause a significant increase of the DOS, although we note a rise below the Fermi level, due to a higher number of states that is a consequence of the higher number of oligomers; however, this does have a significant influence on the band gap of the complex, which becomes more narrow (from $0.34 \mathrm{eV}$ of $\mathrm{P}: \mathrm{F}$ to $0.01 \mathrm{eV}$ of $4 \mathrm{P}: \mathrm{F}$ ), an effect most probably due to an increased level of conjugation of the polymer phase.

We then performed TDDFT, and we required the calculation of the first ten excited states for each one of the complexes. As expected, due to the high number of energetically close orbitals, all the computed excited states of each complex have a charge transfer character, the hole density being always located on the oligomer domain, while the electron density being always found on the fullerene phase. The electron-hole density plots of the $\mathrm{CT}_{1}$ states of $\mathrm{P}: 2 \mathrm{~F}, 2 \mathrm{P}: \mathrm{F}, 3 \mathrm{P}: \mathrm{F}$, and $4 \mathrm{P}: \mathrm{F}$ are shown in Figure 11. The excited state properties of $\mathrm{P}: \mathrm{F}$ have already been discussed in the previous section (see results regarding the PCBM:5A complex), and therefore for the purpose of this study we will focus mainly on the larger 


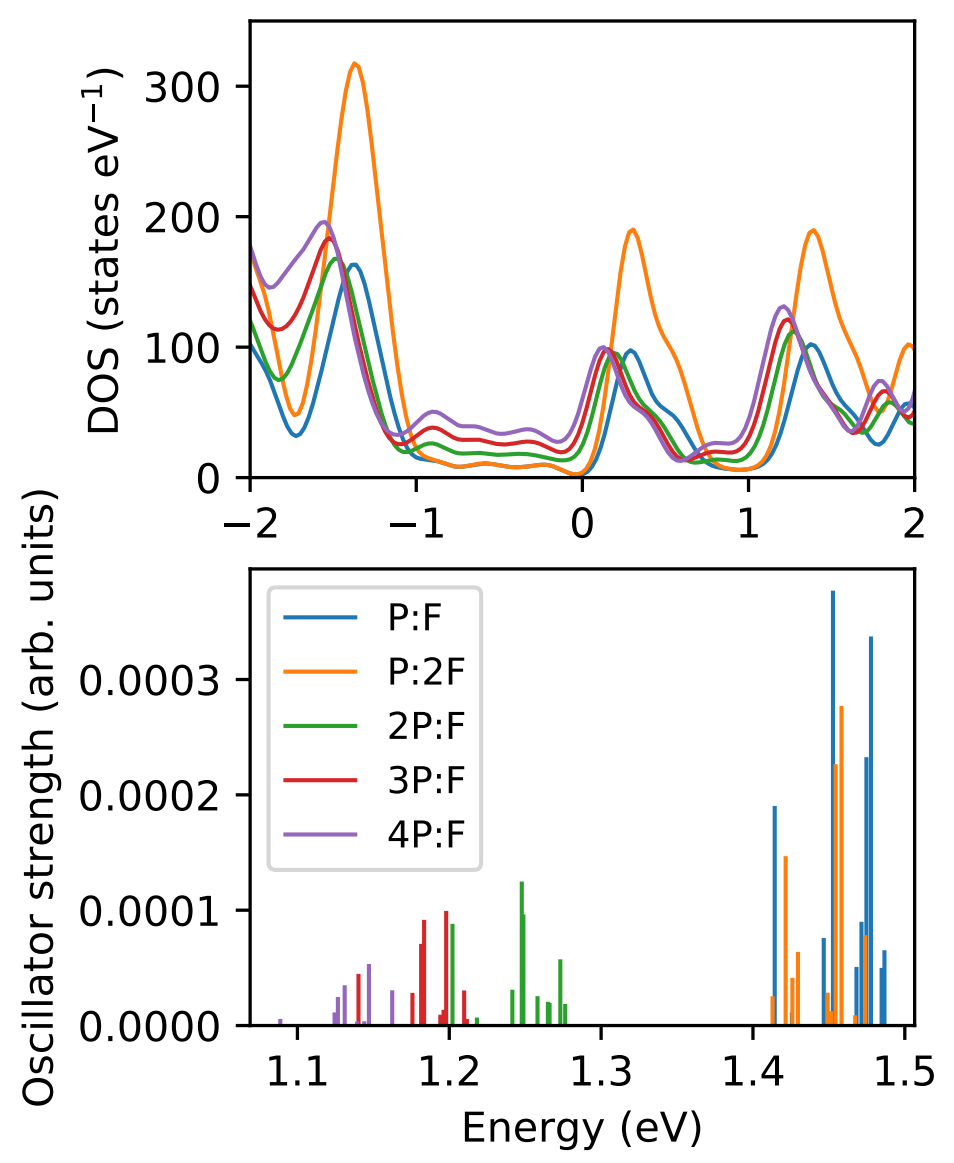

Figure 10: Density of states and excited state spectra of P:F, P:2F, 2P:F, 3P:F, and 4P:F complexes. The DOS are centered around the Fermi levels of the systems, and a Gaussian smearing of $0.1 \mathrm{eV}$ was used. Note that in the spectra a shift was applied to correct the values of the transition energies with respect to experiments. 


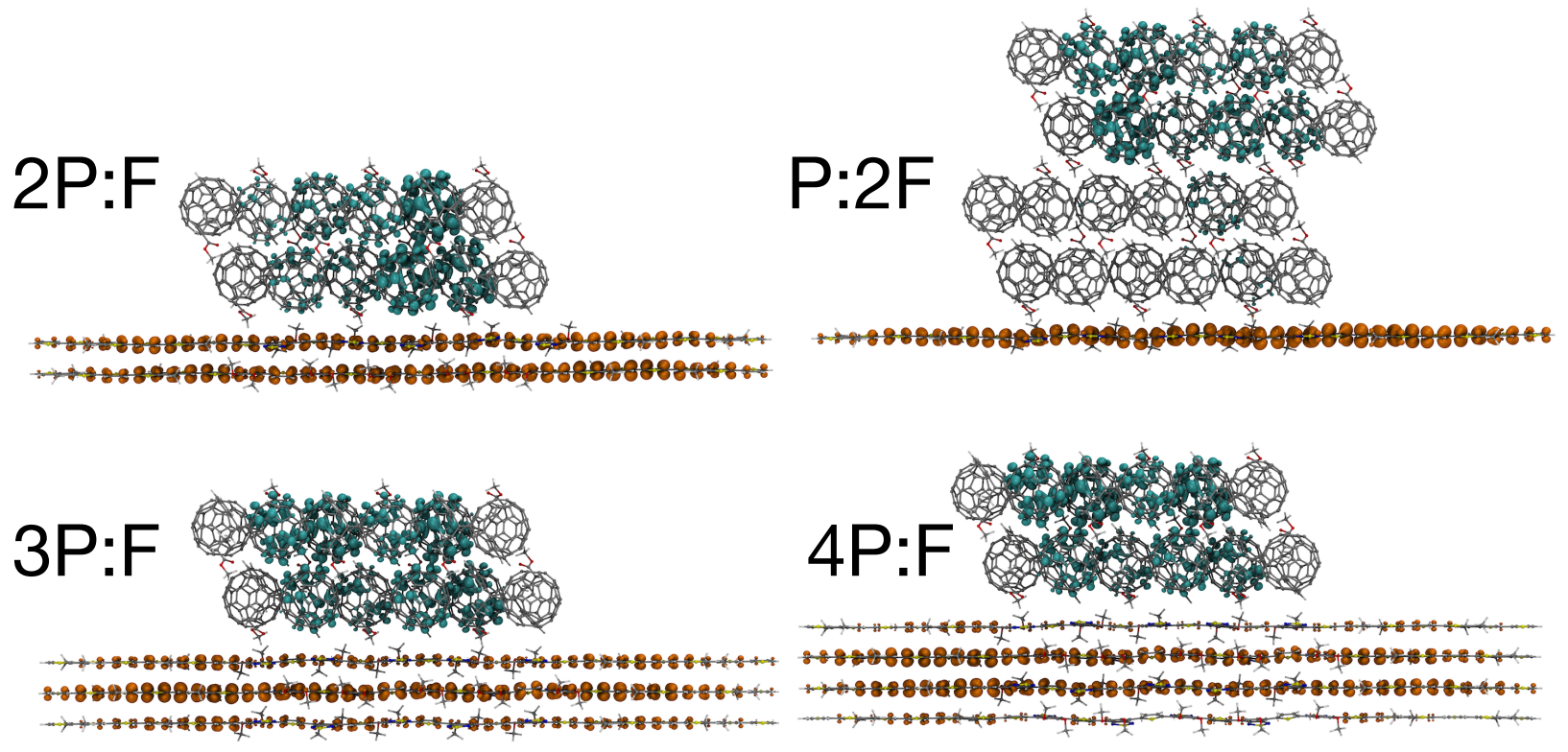

Figure 11: Hole-electron density plots of the $\mathrm{CT}_{1}$ states of P:2F, 2P:F, 3P:F, and 4P:F complexes. See Figure 7 (PCBM:5A) for the density plot of P:F. The electron density is shown in cyan, and the hole density in orange.

complexes. P:2F is particularly interesting because the electron density of its $\mathrm{CT}_{1}$ state extends mostly over the fullerenes which are located far away from the oligomer chain. We estimated the hole-electron separation of this complex by computing the distance between the centroids of the densities, following the procedure described by Le Bahers et al. ${ }^{43} \mathrm{We}$ obtained a value of $\sim 2.8 \mathrm{~nm}$, much higher than $\sim 1 \mathrm{~nm}$, as estimated by Zhang et al. ${ }^{44}$ for short-range $\mathrm{CT}$ states. This confirms that $\mathrm{CT}_{1}$ is a long-range charge transfer state, found in the lowest part of the CT states manifold. This is remarkable, since it is generally believed that CT states with a large electron and hole spatial separation occur at higher energies, and therefore should be regarded as 'hot'. The results here obtained suggest that these states can also have a 'cold' nature. However, the spacing between the excited states of the complexes is really small: for instance, the first three CT states of $\mathrm{P}: 2 \mathrm{~F}$ all lie within $0.02 \mathrm{eV}$, a value smaller than the thermal energy at room temperature $(0.025 \mathrm{eV})$, which makes these states equally thermally accessible. Since the electron densities of these three CT states are located on the PCBM molecules which are both close to and far away from the oligomer, this is further confirmation of the delocalized nature of the electron density, 
and of the possibility of long-range charge transfer processes to occur at lower energies. Similarly, also the electron densities of the 2P:F, 3P:F, and 4P:F complexes are delocalized over the whole fullerene domain, as well as the hole densities over the polymer domain. However, it appears that increasing the number of chains leads to a more localized hole over the inner oligomers of the phase. Overall, these results seem to suggest that: (i) in a bulk heterojunction the charge transfer process does not involve only a single polymer chain and a single PCBM molecule; (ii) long-range charge transfer is possible, and it may occur in the lowest part of the CT state spectrum, in competition with short-range charge transfer; (iii) excited states are generally delocalized over the whole fullerene domain, and over more than a single polymer chain.

Table 2: Breakdown of the first ten CT states of P:F, P:2F, 2P:F, 3P:F, and 4P:F complexes. ${ }^{a}$

\begin{tabular}{|c|c|c|c|c|c|}
\hline CT & $P: F$ & $\mathrm{P}: 2 \mathrm{~F}$ & $2 \mathrm{P}: \mathrm{F}$ & $3 \mathrm{P}: \mathrm{F}$ & $4 \mathrm{P}: \mathrm{F}$ \\
\hline 1 & $\mathrm{HOMO} \rightarrow$ LUMO & $\mathrm{HOMO} \rightarrow$ LUMO & $\mathrm{HOMO} \rightarrow$ LUMO & $\mathrm{HOMO} \rightarrow \mathrm{LUMO}$ & $\mathrm{HOMO} \rightarrow \mathrm{LUMO}$ \\
\hline 2 & $\mathrm{HOMO} \rightarrow \mathrm{LUMO}+1$ & $\mathrm{HOMO} \rightarrow \mathrm{LUMO}+1$ & $\mathrm{HOMO} \rightarrow \mathrm{LUMO}+1$ & $\mathrm{HOMO} \rightarrow \mathrm{LUMO}+1$ & $\mathrm{HOMO} \rightarrow \mathrm{LUMO}+1$ \\
\hline 3 & $\mathrm{HOMO} \rightarrow \mathrm{LUMO}+2$ & $\mathrm{HOMO} \rightarrow \mathrm{LUMO}+2$ & $\mathrm{HOMO} \rightarrow \mathrm{LUMO}+2$ & $\mathrm{HOMO} \rightarrow \mathrm{LUMO}+2$ & $\mathrm{HOMO} \rightarrow \mathrm{LUMO}+2$ \\
\hline 4 & $\mathrm{HOMO} \rightarrow \mathrm{LUMO}+3$ & $\mathrm{HOMO} \rightarrow \mathrm{LUMO}+3$ & $\mathrm{HOMO} \rightarrow \mathrm{LUMO}+3$ & HOMO-1 $\rightarrow$ LUMO & HOMO-1 $\rightarrow$ LUMO \\
\hline 5 & $\mathrm{HOMO} \rightarrow \mathrm{LUMO}+4$ & $\mathrm{HOMO} \rightarrow \mathrm{LUMO}+4$ & HOMO- $1 \rightarrow$ LUMO & $\mathrm{HOMO} \rightarrow \mathrm{LUMO}+3$ & $\mathrm{HOMO} \rightarrow \mathrm{LUMO}+3$ \\
\hline 6 & $\mathrm{HOMO} \rightarrow \mathrm{LUMO}+5$ & $\mathrm{HOMO} \rightarrow \mathrm{LUMO}+5$ & $\mathrm{HOMO} \rightarrow \mathrm{LUMO}+4$ & $\mathrm{HOMO}-1 \rightarrow \mathrm{LUMO}+1$ & HOMO- $1 \rightarrow$ LUMO +1 \\
\hline 7 & HOMO-1 $\rightarrow$ LUMO & $\mathrm{HOMO} \rightarrow \mathrm{LUMO}+6$ & HOMO-1 $\rightarrow$ LUMO +1 & $\mathrm{HOMO} \rightarrow \mathrm{LUMO}+4$ & $\mathrm{HOMO} \rightarrow \mathrm{LUMO}+4$ \\
\hline 8 & $\mathrm{HOMO} \rightarrow \mathrm{LUMO}+6$ & $\mathrm{HOMO} \rightarrow \mathrm{LUMO}+7$ & $\mathrm{HOMO} \rightarrow \mathrm{LUMO}+5$ & $\mathrm{HOMO} \rightarrow \mathrm{LUMO}+5$ & $\mathrm{HOMO} \rightarrow \mathrm{LUMO}+5$ \\
\hline 9 & $\mathrm{HOMO} \rightarrow \mathrm{LUMO}+7$ & $\begin{array}{l}\mathrm{HOMO} \rightarrow \mathrm{LUMO}+8 \\
014(\mathrm{HOMO} \rightarrow \mathrm{LUM}\end{array}$ & $\mathrm{HOMO} \rightarrow \mathrm{LUMO}+6$ & $\mathrm{HOMO} \rightarrow \mathrm{LUMO}+6$ & $\begin{array}{l}0.84(\mathrm{HOMO} \rightarrow \mathrm{LUMO}+6) \\
0.15(\mathrm{HOMO} \rightarrow \mathrm{LUMO}+7)\end{array}$ \\
\hline 10 & $\mathrm{HOMO}-1 \rightarrow \mathrm{LUMO}+1$ & $\begin{array}{l}0.70(\mathrm{HOMO} \rightarrow \mathrm{LUMO}+10) \\
0.14(\mathrm{HOMO} \rightarrow \mathrm{LUMO}+11) \\
0.10(\mathrm{HOMO}-1 \rightarrow \mathrm{LUMO})\end{array}$ & $\mathrm{HOMO} \rightarrow \mathrm{LUMO}+7$ & $\mathrm{HOMO} \rightarrow \mathrm{LUMO}+7$ & $\mathrm{HOMO}-1 \rightarrow \mathrm{LUMO}+2$ \\
\hline
\end{tabular}

${ }^{a}$ Note that every transition has a charge transfer character, and therefore every electron-donating orbital is located on the oligomer while every electron-accepting orbital is located on the fullerene domain.

A breakdown and a plot of the computed CT states are shown in Table 2 and Figure 10, lower plot, respectively. Multiplying the polymer and the fullerene phases does influence the character of the $\mathrm{CT}$ states: for instance, while $\mathrm{P}: \mathrm{F}, 2 \mathrm{P}: \mathrm{F}, 3 \mathrm{P}: \mathrm{F}$, and $4 \mathrm{P}: \mathrm{F}$ all present transitions from both the HOMO and HOMO-1 levels of the polymer domain, the CT states of $\mathrm{P}: 2 \mathrm{~F}$ involve almost exclusively transitions from the HOMO of the oligomer to the virtual orbitals of the fullerene domain; only in $\mathrm{CT}_{10} \mathrm{HOMO}-1$ does take part, even if only with a small contribution, to the excited state transition. The absorption spectra are also significantly different, as increasing the size of the polymer domain leads to the lowering of both 
the energies and the intensities of the transitions, as can be observed in Figure 10, lower plot. The decrease of the transition energies reflects the decrease of the band gap of the complex, as previously discussed, while the decrease of the oscillator strengths can be explained with a reduced coupling between the densities of the fullerene and the polymer domain, the latter progressively moving onto the inner chains of the phase, this causing a poor overlap with the electron density. We also note here that low oscillator strengths are beneficial for the population of CT states, since this process is more likely to happen via internal conversion from the polymer bright $S_{1}$ state rather than via direct excitation. Increasing the fullerene phase does not affect the range of energies at which the CT states occur, suggesting small or no relation between the size of the PCBM domain and the energy of the CT states. As expected, we also observe a smaller spacing between the excited states and consequently an increased number of states within the same energy range. We then also computed $E_{\mathrm{B}}^{\mathrm{CT}}$ for each one of the complexes, and as expected, due to the higher delocalization of in turn the hole and the electron densities, we observed smaller values when multiplying the polymer and the fullerene domains: the computed charge transfer exciton binding energy of $\mathrm{P}: \mathrm{F}$ is $0.68 \mathrm{eV}$, which then becomes $0.51 \mathrm{eV}$ for $\mathrm{P}: 2 \mathrm{~F}$, and $0.60 \mathrm{eV}, 0.50 \mathrm{eV}$, and $0.49 \mathrm{eV}$ for $2 \mathrm{P}: \mathrm{F}$, 3P:F, and 4P:F, respectively. As it can be observed, $E_{\mathrm{B}}^{\mathrm{CT}}$ converges to $\sim 0.5 \mathrm{eV}$, a value which is compatible with experimental estimates of CT exciton binding energies present in literature, and made on a significant number of polymer-fullerene blends: $0.1-0.5 \mathrm{eV} \cdot{ }^{36,45,46}$ Although increasing the size of both phases seems to be beneficial, we do note that $E_{\mathrm{B}}^{\mathrm{CT}}$ presents a stronger decrease when the polymer domain is multiplied, suggesting that a large polymer phase may significantly facilitate the hole-electron splitting rather than a large fullerene phase. 


\section{Conclusion}

There is still some lack of understanding of the mechanisms that govern the photoinduced charge-carrier formation in bulk heterojunction devices. Due to their complex morphologies, it is not trivial to construct theoretical models that are representative of a real device, one of the main problems being the computational cost associated with density functional calculations on large systems such as polymer-fullerene complexes. By using a linear-scaling reformulation of DFT within the ONETEP code, we were able to overcome the size issue and perform fully ab initio calculations on a number of large-scale polymer-fullerene blends, with the aim of exploring some of the ground- and excited-state properties relevant for OPV functionality. Ground-state properties were investigated by performing DFT calculations, while to study excited-state properties we used time-dependent DFT (TDDFT).

We first explored the effect of different block lengths of the polymer chain when interacting with the fullerene domain, with a focus on the size of the A block. Despite observing no significant changes in the ground-state properties, we did note some differences in the excitedstate spectra of these complexes: we found that the size of the oligomers' A block correlates with the average intensity of the charge transfer states, that is, the larger the extent of the A block, the stronger the oscillator strength. By decoupling the oligomer from the PCBM phase, we were able to observe that the electron density of the oligomers $\mathrm{S}_{1}$ transition is found exclusively above the A blocks: therefore, large A blocks enhance the coupling between the oligomer electron density and the fullerene crystal, increasing the probability of a charge transfer to occur. By treating the fullerene phase as a close-packed crystal, we were also able to observe a delocalized electron density, suggesting that during the charge transfer more than a single PCBM molecule is able to interact at the same time with the polymer. We also computed the charge transfer exciton binding energies, and we observed that the complex composed of the oligomer with the shortest A block had the highest $E_{\mathrm{B}}^{\mathrm{CT}}(0.71 \mathrm{eV})$, while the lowest value $(0.61 \mathrm{eV})$ was found for the complex composed of the alternating A-D oligomer; both complexes, however, were characterized by similar and low oscillator strengths. These 
results suggest that the size of the polymer A block does influence the excited state manifold of a BHJ blend, and that, in order to maximize the probability of a CT transition to occur, a polymer with large acceptor blocks may be beneficial.

We then examined the effect of both the fullerene and the polymer domains size, by selectively increasing the size of one phase with respect to the other. When we doubled the fullerene phase (from 12 to 24 PCBM molecules) we could not observe any significant difference in the energy range of the CT states with respect to our starting model; we did observe, however, an increase in the number of $\mathrm{CT}$ states, due to the higher number of molecules in the complex. The electron densities of the $\mathrm{CT}$ transitions all have a strongly delocalized character, involving fullerenes both close to and far away from the oligomer chain; some of the lowest states (for instance, the first one, $\mathrm{CT}_{1}$ ) are characterized by an electron density found almost exclusively on the PCBM molecules positioned at the upper edge of the complex, with a hole-electron separation of $\sim 2.8 \mathrm{~nm}$; this suggests that long-range charge transfer states are possible, and that they can be observed even at the lowest energies. These long-range CT states most probably coexist with short-range states, since the energy difference of both $\mathrm{CT}_{2}$ and $\mathrm{CT}_{3}$ with respect to $\mathrm{CT}_{1}$ is less than the thermal energy at room temperature. We then progressively increased the number of oligomer chains (from 1 to 4) while keeping the fullerene phase constant, and we observed a decrease of the CT state energies, which correlates with the number of oligomer chains. Lower oscillator strengths also correlate with a higher number of chains, and this can be explained by examining the hole densities of the complexes, which, like the electron densities, show a delocalized character over more than a single polymer: the higher the number of chains and the more the hole density moves into the inner chains of the polymer phase, reducing the coupling with the fullerene phase. We finally computed the charge transfer exciton binding energies and we observed that increasing the polymer phase significantly lowers the value of $E_{\mathrm{B}}^{\mathrm{CT}}$, while increasing the PCBM domain has a less strong effect on $E_{\mathrm{B}}^{\mathrm{CT}}$, despite also causing a lowering of the value. 
Clearly, given the complexity of the problem, only a limited number of questions were addressed here; nevertheless, we expect the results obtained in this paper to be helpful for the design of high-performance OPV devices, as we were able to shed light into some of the charge generation mechanisms of a polymer-fullerene BHJ.

\section{Acknowledgement}

G. Boschetto thanks both Merck and EPSRC for supporting his Ph.D. studentship via a Doctoral Training Centre Grant (no. EP/L015382/1). All the calculations were carried out on the Iridis4 Supercomputer of the University of Southampton.

\section{Supporting Information Available}

Excited state properties of isolated blend components. Effect of relative positioning of PCBM with respect to D and A units of one polymer repeat unit and a long chain oligomer.

\section{References}

(1) Günes, S.; Neugebauer, H.; Sariciftci, N. S. Conjugated Polymer-Based Organic Solar Cells. Chem. Rev. 2007, 107, 1324-1338.

(2) Brabec, C. J.; Gowrisanker, S.; Halls, J. J. M.; Laird, D.; Jia, S.; Williams, S. P. Polymer-Fullerene Bulk-Heterojunction Solar Cells. Adv. Mater. 2010, 22, 3839-3856.

(3) Yu, G.; Gao, J.; Hummelen, J. C.; Wudl, F.; Heeger, A. J. Polymer Photovoltaic Cells : Enhanced Efficiencies via a Network of Internal Donor-Acceptor Heterojunctions. Science 1995, 270, 1789-1791.

(4) Pensack, R. D.; Asbury, J. B. Barrierless Free Carrier Formation in an Organic Photo- 
voltaic Material Measured with Ultrafast Vibrational Spectroscopy. J. Am. Chem. Soc. 2009, 131, 15986-15987.

(5) Zhu, X.-Y.; Yang, Q.; Muntwiler, M. Charge-Transfer Excitons at Organic Semiconductor Surfaces and Interfaces. Acc. Chem. Res. 2009, 42, 1779-1787.

(6) Ohkita, H.; Cook, S.; Astuti, Y.; Duffy, W.; Tierney, S.; Zhang, W.; Heeney, M.; McCulloch, I.; Nelson, J.; Bradley, D. D. C. et al. Charge Carrier Formation in Polythiophene/Fullerene Blend Films Studied by Transient Absorption Spectroscopy. J. Am. Chem. Soc. 2008, 130, 3030-3042.

(7) Shoaee, S.; Clarke, T. M.; Huang, C.; Barlow, S.; Marder, S. R.; Heeney, M.; McCulloch, I.; Durrant, J. R. Acceptor Energy Level Control of Charge Photogeneration in Organic Donor/Acceptor Blends. J. Am. Chem. Soc. 2010, 132, 12919-12926.

(8) Lee, J.; Vandewal, K.; Yost, S. R.; Bahlke, M. E.; Goris, L.; Baldo, M. A.; Manca, J. V.; Van Voorhis, T. Charge Transfer State Versus Hot Exciton Dissociation in PolymerFullerene Blended Solar Cells. J. Am. Chem. Soc. 2010, 132, 11878-11880.

(9) van der Hofstad, T. G. J.; Di Nuzzo, D.; van den Berg, M.; Janssen, R. A. J.; Meskers, S. C. J. Influence of Photon Excess Energy on Charge Carrier Dynamics in a PolymerFullerene Solar Cell. Adv. Energy Mater. 2012, 2, 1095-1099.

(10) Vandewal, K.; Albrecht, S.; Hoke, E. T.; Graham, K. R.; Widmer, J.; Douglas, J. D.; Schubert, M.; Mateker, W. R.; Bloking, J. T.; Burkhard, G. F. et al. Efficient Charge Generation by Relaxed Charge-Transfer States at Organic Interfaces. Nat. Mater. 2014, $13,63-68$.

(11) Liu, Y.; Zhao, J.; Li, Z.; Mu, C.; Ma, W.; Hu, H.; Jiang, K.; Lin, H.; Ade, H.; Yan, H. Aggregation and Morphology Control Enables Multiple Cases of High-Efficiency Polymer Solar Cells. Nat. Commun. 2014, 5, 5293. 
(12) Chen, J.-D.; Cui, C.; Li, Y.-Q.; Zhou, L.; Ou, Q.-D.; Li, C.; Li, Y.; Tang, J.-X. SingleJunction Polymer Solar Cells Exceeding 10\% Power Conversion Efficiency. Adv. Mater. 2015, 27, 1035-1041.

(13) He, Z.; Xiao, B.; Liu, F.; Wu, H.; Yang, Y.; Xiao, S.; Wang, C.; Russell, T. P.; Cao, Y. Single-Junction Polymer Solar Cells With High Efficiency and Photovoltage. Nat. Photonics 2015, 9, 174-179.

(14) Few, S.; Frost, J. M.; Kirkpatrick, J.; Nelson, J. Influence of Chemical Structure on the Charge Transfer State Spectrum of a Polymer:Fullerene Complex. J. Phys. Chem. C 2014, 118, 8253-8261.

(15) Niedzialek, D.; Duchemin, I.; Branquinho de Queiroz, T.; Osella, S.; Rao, A.; Friend, R.; Blase, X.; Kümmel, S.; Beljonne, D. First Principles Calculations of Charge Transfer Excitations in Polymer-Fullerene Complexes: Influence of Excess Energy. Adv. Funct. Mater. 2015, 25, 1972-1984.

(16) Falke, S. M.; Rozzi, C. A.; Brida, D.; Maiuri, M.; Amato, M.; Sommer, E.; De Sio, A.; Rubio, A.; Cerullo, G.; Molinari, E. et al. Coherent Ultrafast Charge Transfer in an Organic Photovoltaic Blend. Science 2014, 344, 1001-1005.

(17) Fazzi, D.; Barbatti, M.; Thiel, W. Hot and Cold Charge-Transfer Mechanisms in Organic Photovoltaics: Insights into the Excited States of Donor/Acceptor Interfaces. J. Phys. Chem. Lett. 2017, 8, 4727-4734.

(18) Berny, S.; Blouin, N.; Distler, A.; Egelhaaf, H.-J.; Krompiec, M.; Lohr, A.; Lozman, O. R.; Morse, G. E.; Nanson, L.; Pron, A. et al. Solar Trees: First Large-Scale Demonstration of Fully Solution Coated, Semitransparent, Flexible Organic Photovoltaic Modules. Adv. Sci. 2016, 3, 1500342.

(19) Menelaou, C.; Tierney, S.; Blouin, N.; Mitchell, W.; Tiwana, P.; McKerracher, I.; Jagadish, C.; Carrasco, M.; Herz, L. M. Effect of Nanocrystalline Domains in Photovoltaic 
Devices With Benzodithiophene-Based Donor-Acceptor Copolymers. J. Phys. Chem. C 2014, 118, 17351-17361.

(20) Ma, H.; Troisi, A. Direct Optical Generation of Long-Range Charge-Transfer States in Organic Photovoltaics. Adv. Mater. 2014, 26, 6163-6167.

(21) Skylaris, C.-K.; Haynes, P. D.; Mostofi, A. A.; Payne, M. C. Introducing ONETEP: Linear-Scaling Density Functional Simulations on Parallel Computers. J. Chem. Phys. 2005, 122, 084119.

(22) Skylaris, C.-K.; Mostofi, A. A.; Haynes, P. D.; Diéguez, O.; Payne, M. C. Nonorthogonal Generalized Wannier Function Pseudopotential Plane-Wave Method. Phys. Rev. B 2002, 66, 035119.

(23) Mostofi, A. A.; Haynes, P. D.; Skylaris, C.-K.; Payne, M. C. Preconditioned iterative minimization for linear-scaling electronic structure calculations. J. Chem. Phys 2003, 119,8842 .

(24) Paternò, G.; Warren, A. J.; Spencer, J.; Evans, G.; Sakai, V. G.; Blumberger, J.; Cacialli, F. Micro-Focused X-ray Diffraction Characterization of High-Quality [6,6]Phenyl- $\mathrm{C}_{61}$-Butyric Acid Methyl Ester Single Crystals Without Solvent Impurities. J. Mater. Chem. C 2013, 1, 5619-5623.

(25) Borges, I. J.; Aquino, A. J. A.; Köhn, A.; Nieman, R.; Hase, W. L.; Chen, L. X.; Lischka, H. Ab Initio Modeling of Excitonic and Charge-Transfer States in Organic Semicondictors: The PTB1/PCBM Low Band Gap System. J. Am. Chem. Soc. 2013, $135,18252-18255$.

(26) Perdew, J. P.; Burke, K.; Ernzerhof, M. Generalized Gradient Approximation Made Simple. Phys. Rev. Lett. 1996, 77, 3865-3868. 
(27) Hine, N. D. M. Linear-Scaling Density Functional Theory Using the Projector Augmented Wave Method. J. Phys.: Condens. Matter 2017, 29, 024001.

(28) Grimme, S. Semiempirical GGA-Type Density Functional Constructed with a LongRange Dispersion Correction. J. Comput. Chem. 2006, 27, 1787-1799.

(29) Zuehlsdorff, T. J.; Hine, N. D. M.; Spencer, J. S.; Harrison, N. M.; Riley, D. J.; Haynes, P. D. Linear-Scaling Time-Dependent Density-Functional Theory in the Linear Response Formalism. J. Chem. Phys. 2013, 139, 064104.

(30) Zuehlsdorff, T. J.; Hine, N. D. M.; Payne, M. C.; Haynes, P. D. Linear-Scaling TimeDependent Density-Functional Theory Beyond the Tamm-Dancoff Approximation: Obtaining Efficiency and Accuracy with in situ Optimised Local Orbitals. J. Chem. Phys. 2015, 143, 204107.

(31) Hirata, S.; Head-Gordon, M. Time-Dependent Density Functional Theory Within the Tamm-Dancoff Approximation. Chem. Phys. Lett. 1999, 314, 291-299.

(32) Dreuw, A.; Head-Gordon, M. Single-Reference ab Initio Methods for the Calculation of Excited States of Large Molecules. Chem. Rev. 2005, 105, 4009-4037.

(33) Ratcliff, L.; Hine, N. D. M.; Haynes, P. D. Calculating Optical Absorption Spectra for Large Systems Using Linear-Scaling Density Functional Theory. Phys. Rev. B 2011, $84,165131$.

(34) Yao, J.; Kirchartz, T.; Vezie, M. S.; Faist, M. A.; Gong, W.; He, Z.; Wu, H.; Troughton, J.; Watson, T.; Bryant, D. et al. Quantifying Losses in Open-Circuit Voltage in Solution-Processable Solar Cells. Phys. Rev. Applied 2015, 4, 014020.

(35) Benduhn, J.; Tvingstedt, K.; Piersimoni, F.; Ullbrich, S.; Fan, Y.; Tropiano, M.; McGarry, K. A.; Zeika, O.; Riede, M. K.; Douglas, C. J. et al. Intrinsic Non-Radiative Voltage Losses in Fullerene-Based Organic Solar Cells. Nat. Energy 2017, 2, 17053. 
(36) Clarke, T. M.; Durrant, J. R. Charge Photogeneration in Organic Solar Cells. Chem. Rev. 2010, 110, 6736-6767.

(37) Brédas, J.-L.; Norton, J. E.; Cornil, J.; Coropceanu, V. Molecular Understanding of Organic Solar Cells: The Challenges. Acc. Chem. Res. 2009, 42, 1691-1699.

(38) Kurpiers, J.; Ferron, T.; Roland, S.; Jakoby, M.; Thiede, T.; Jaiser, F.; Albrecht, S.; Janietz, S.; Collins, B. A.; Howard, I. A. et al. Probing the Pathways of Free Charge Generation in Organic Bulk Heterojunction Solar Cells. Nat. Commun. 2018, 9, 2038.

(39) Ravva, M. K.; Wang, T.; Brédas, J.-L. Nature of the Binding Interactions Between Conjugated Polymer Chains and Fullerenes in Bulk Heterojunction Organic Cells. Chem. Mater. 2016, 28, 8181-8189.

(40) Lawton, S. Investigating the Structure-Property Relationship of Donor-Acceptor Type Polymers in Organic Solar Cells. Presented at the 23rd annual Merck CASE Conference, Brockenhurst, United Kingdom, 2017.

(41) Slama-Schwok, A.; Blanchard-Desce, M.; Lehn, J. M. Intramolecular Charge Transfer in Donor-Acceptor Molecules. J. Phys. Chem. 1990, 94, 3894-3902.

(42) Blanchard-Desce, M.; Wortmann, R.; Lebus, S.; Lehn, J.-M.; Krämer, P. Intramolecular Charge Transfer in Elongated Donor-Acceptor Conjugated Polyenes. Chem. Phys. Lett. 1995, 243, 526-532.

(43) Le Bahers, T.; Adamo, C.; Ciofini, I. A Qualitative Index of Spatial Extent in ChargeTransfer Excitations. J. Chem. Theory Comput. 2011, 7, 2498-2506.

(44) Zhang, J.; Jakowetz, A. C.; Li, G.; Di, D.; Matthew Menke, S.; Rao, A.; Friend, R. H.; Bakulin, A. A. On the Energetics of Bound Charge-Transfer States in Organic Photovoltaics. J. Mater. Chem. A 2007, 5, 11949. 
(45) Veldman, D.; Meskers, S. C. J.; Janssen, R. A. J. The Energy of Charge-Transfer States in Electron Donor-Acceptor Blends: Insight into the Energy Losses in Organic Solar Cells. Adv. Funct. Mater. 2009, 19, 1939-1948.

(46) Blom, P. W. M.; Mihailetchi, V. D.; Jan Anton Koster, L.; Markov, D. E. Device Physics of Polymer:Fullerene Bulk Heterojunction Solar Cells. Adv. Mater. 2007, 19, $1551-1566$. 


\section{Graphical TOC Entry}

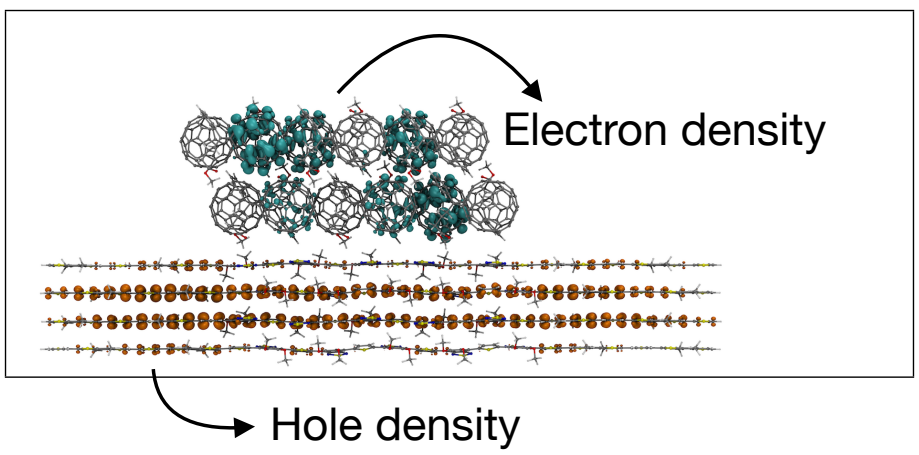

